\title{
The Research on Ocean Surface Wind Speed Retrieval by Neural Network Algorithm for HY2 Altimeter
}

\author{
Jiasheng Tian*, Hang Xue, Jian Shi \\ School of Electronic Information and Communications, Huazhong University of Science and Technology, Wuhan \\ city, Hubei Province, China \\ Email: tianjs@hust.edu.cn
}

\begin{abstract}
The neural network algorithm in this paper is applied to the ocean surface wind speed retrievals. Firstly, the Ku band backscattering coefficient $\left(\sigma_{\mathrm{Ku}}^{0}\right)$ is considered as the input parameter to retrieve the wind speed and the retrieval precision reaches $1 \mathrm{~m} / \mathrm{s}$ (root mean square error) for HY2 altimeter. Secondly, by introducing the Ku-band significant wave height $\left(\mathrm{swh}_{\mathrm{ku}}\right)$, C-band backscattering coefficients $\left(\sigma_{\mathrm{C}}^{0}\right)$ and C-band $\mathrm{swh}\left(\mathrm{swh}_{\mathrm{C}}\right)$ as input parameters to inverse wind speed, the retrieved results show that the multi-parameter algorithm introduced in the neural network can effectively improve the wind speed retrieval accuracy. The wind speed is not only relative to $\sigma_{\text {Ku }}^{0}$, but also to $\sigma_{C}^{0}, s w h_{k u}$ and $\mathrm{swh}_{\mathrm{C}}$. The neural networks algorithm is available for HY2 altimeter wind speed retrieval.
\end{abstract}

Keywords: Neural network, wind speed retrieval, backscatter coefficient, multi-parameter algorithm.

\section{Introduction}

Satellite altimeter transmits radio waves to ocean surface and receives it. By extracting information from echo signal we can derive the dynamic topography and parameters of the oceans such as swh and sea surface wind speed. The accurate inversion of ocean surface wind speed plays a very important role in human development and utilization of Marine resources. Compared with swh and sea surface height (ssh) the wind speed retrieval accuracy is not satifying by using a single parameter $\sigma_{K u}^{0}$. In order to improve the wind speed inversion, many efforts have been carried out recently [3-6].

The HY-2 satellite was successfully launched on 16 August 2011. It carried four microwave instruments for observing dynamic ocean environment parameters on a global scale. In this paper Neural network will be applied to HY2 altimeter wind speed retrieval. Neural network is a processing technology similar to the human nervous system with self-learning and adaptive, nonlinear approximation capability and the characteristics of distributed storage. Without any predefined mathematical model, the weight adjustment by error back propagation can be effective to establish the relationship between the inputs and outputs.

According to the data from HY2 satellite altimeter, three models were established by neural network: (1) neural network single parameter inversion: $\sigma_{K u}^{0}$ for wind speed retrievals; (2)Neural network dualparameters inversion: $\sigma_{K u}^{0}$ and Ku-band $s w h_{k u}$ for wind speed retrievals;(3) Neural network multiparameter inversion: $\sigma_{K u}^{0}, \sigma_{C}^{0}$ and $\operatorname{swh}\left(s w h_{k u}, s w h_{C}\right)$ for wind speed retrievals. The results show that neural network algorithm is suitable for HY2 altimeter wind speed, and also has a good retrieval accuracy especially by introducing $\sigma_{C}^{0}, s w h_{k u}$ and $s w h_{C}$.

\section{The Basic Principle of Neural Network}

Feedforward neural networks propagate the inputs through a set of computational nodes arranged in layers to calculate the network outputs. The output layer is the final layer of the neural network and usually contains linear elements. The layers between the input layer and the output layer are called hidden layers and usually contain nonlinear elements. The various types of feedforward neural networks 
differ primarily in the nonlinear functions (the so-called activation functions) that are used in the hidden layer nodes and the training algorithms that are used to optimize the free parameters of the network.

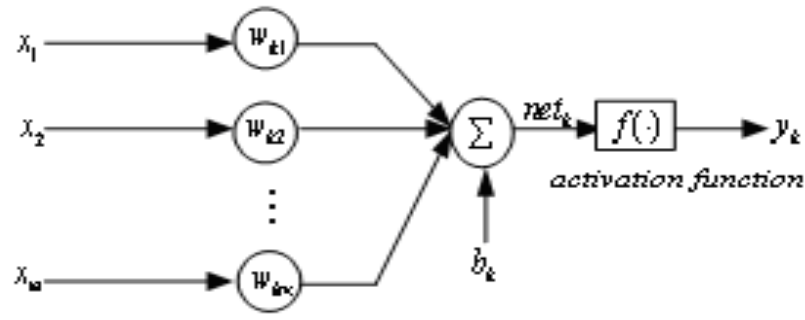

Figure 2.1. Perceptron Structure

The perceptron is the basic structural element of feedforward multilayer perceptron networks. The perceptron is shown graphically in Figure 2.1. The transfer function can be given as

$$
n e t_{k}=\sum_{j=1}^{m} W_{k j} \cdot x_{j}+b_{k}
$$

where $y_{k}=f(n e t)$ and $x_{j}$ is the $j$ th input, $W_{k j}$ is the weight associated with the $j$ th input, $b_{\mathrm{k}}$ is the bias, $f($. is the activation function of the perceptron, and $y_{k}$ is the output. The activation function in hidden layer can be given as

$$
f(x)=\frac{1}{1+e^{-x}}
$$

while the activation function in output layer is

$$
f(x)=x
$$

The network includes multiple layers which have a good number of neurons. The input layer is only used to receive input signal, and does not involve calculating; The hidden layer has many more complex patterns which can be executed and identified. We can get inversion results from the output layer. Theory and practices show that a three-layer BP network as shown in figure 2.2, can achieve arbitrary continuous map, and also approximate arbitrary given continuous function by increasing the hidden layer nodes to improve accuracy.
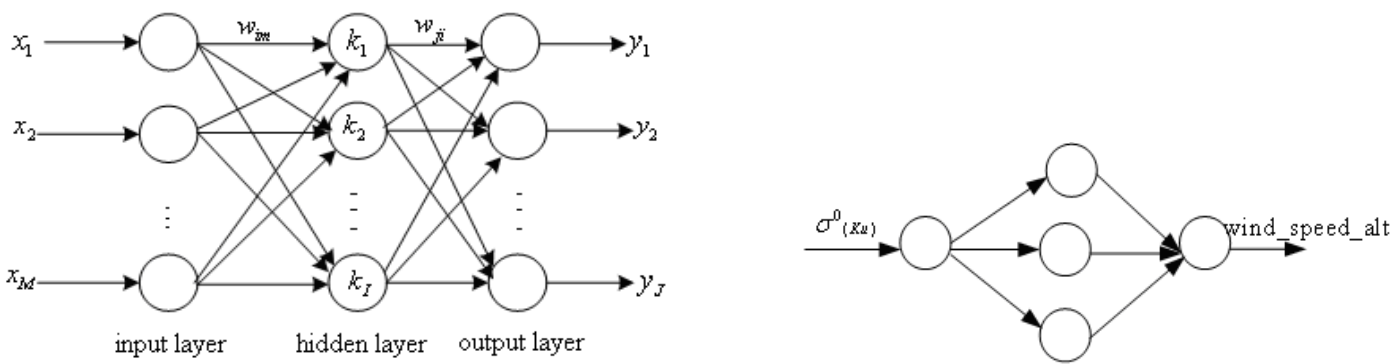

Figure 2.2. Three layers structure

Figure 3.1. Neural network structure

\section{Ocean Surface Wind Speed Inversion}

Some data about $\sigma_{K u}^{0}, \sigma_{C}^{0}, s w h_{k u}$ and $s w h_{C}$ and sea surface wind speed from the 2015 annual HY-2 altimeter are extracted, and the abnormal records are excluded. The structure of the training network is shown in Figure 3.1. The training and retrieval are done by changing the input parameters. 


\subsection{Using $\sigma^{0} \mathrm{Ku}$ to Retrieve Wind Speed}

In terms of file H2ARA1IDR2PT009000272015030100390720150301013112.nc, some parameters are read. $\sigma_{K u}^{0}$ is used as input parameter and wind speed as output parameter. We choose the data in period 00:39:07 01:31:12 of day 2015/03/01 to obtain network parameters. We use the first 1000 sets of data as training samples and the rest as testing samples. We compare the tested values with those measured ones by calculating the corresponding root mean square error RMSE.

Those measurements by HY2 and corresponding retrieval values are plotted in Figure 3.2. The root mean square error RMSE is $1.0564 \mathrm{~cm} / \mathrm{s}$.

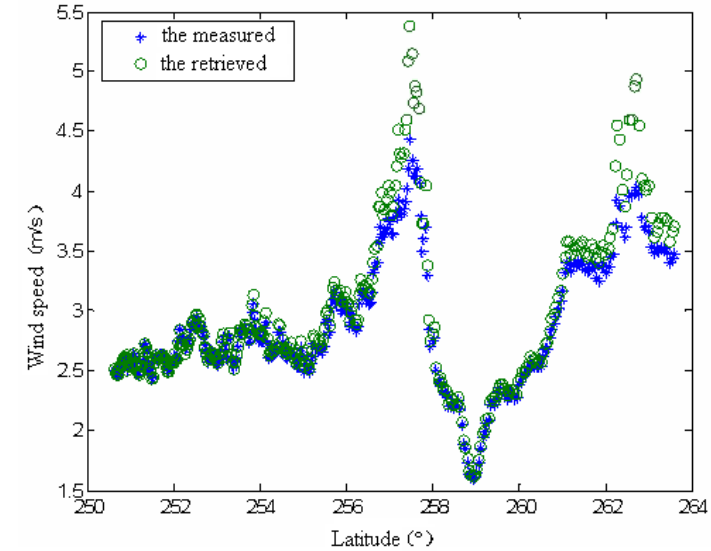

Figure $\quad 3.2 \quad$ Measurements and retrieval values retrieval values

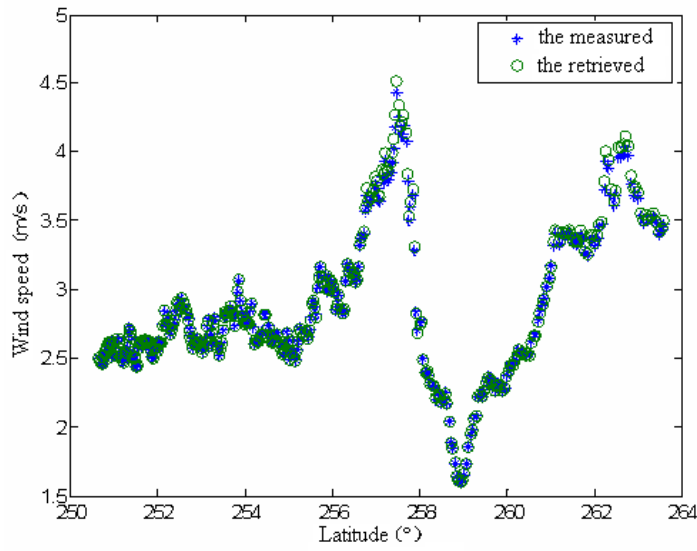

Figure3.3 Measurements and

We have also used some data from other time periods as test data to study the wind speed retrieval model. The results are shown as follows in Table 3.1.

Table 3.1 The root mean square error value for other test data

\begin{tabular}{|c|c|c|c|}
\hline training sample & \multicolumn{3}{|c|}{ 2015/03/01 00:39:07 01:31:12 } \\
\hline \multicolumn{3}{|l|}{ test data } & $\operatorname{RMSE}(\mathrm{cm} / \mathrm{s})$ \\
\hline DATA1: The same time & $2015 / 03 / 01$ & 00:39:07 01:31:12 & 1.0564 \\
\hline \multirow[t]{3}{*}{ DATA2: Different time } & $2015 / 03 / 14$ & 02:01:17 02:53:12 & 22.2596 \\
\hline & $2015 / 04 / 25$ & 02:01:29 02:53:41 & 13.9843 \\
\hline & $2014 / 04 / 12$ & $01: 59: 14 \sim 02: 51: 28$ & 72.8879 \\
\hline \multirow[t]{3}{*}{ DATA3: Different month or different time } & $2015 / 03 / 14$ & $13: 20: 25 \sim 14: 12: 28$ & 50.8262 \\
\hline & $2015 / 09 / 26$ & $02: 02: 06 \sim 02: 54: 18$ & 27.6224 \\
\hline & $2015 / 12 / 25$ & 02:02:18 02:54:11 & 114.9361 \\
\hline
\end{tabular}

For data2 and data3, the mean RMSE is $50.4194 \mathrm{~cm} / \mathrm{s}$, the range is from 13.9843 to 114.9361 . It is obvious that a single parameter is used to retrieve the wind speed, the root mean square error is large and also unstable.

\subsection{Using $\sigma_{\mathrm{Ku}}^{0}$ and $\mathrm{Ku}$-Band SWH to Retrieve the Wind Speed}

Using the same method described above, we use $\sigma_{K u}^{0}$ and Ku-band $s w h_{k u}$ as input parameters and wind speed as output parameter to obtain network parameters. We choose the data in period (00:39:07 01:31:12) of 2015/03/01, and use the first 1000 sets of data as training samples and the rest as testing samples. Compare the tested and measured values by calculating RMSE, as shown in figure 3.3. We 
have also used some data from other time periods to do the retrieval. The results are shown as follows in table 3.2 .

Table 3.2 The root mean square error value for other test data

\begin{tabular}{|l|c|c|c|}
\hline training sample & \multicolumn{3}{|c|}{$2015 / 03 / 01 \quad 00: 39: 07 \sim 01: 31: 12$} \\
\hline \multicolumn{3}{|c|}{ test data } & RMSE $(\mathrm{cm} / \mathrm{s})$ \\
\hline \multirow{2}{*}{$\begin{array}{l}\text { DATA1: } \\
\text { The same time }\end{array}$} & $2015 / 03 / 01$ & $00: 39: 07 \sim 01: 31: 12$ & 0.4778 \\
\hline \multirow{2}{*}{$\begin{array}{l}\text { DATA2: } \\
\text { Different time }\end{array}$} & $2015 / 03 / 14$ & $02: 01: 17 \sim 02: 53: 12$ & 1.3525 \\
\cline { 2 - 4 } & $2015 / 04 / 25$ & $02: 01: 29 \sim 02: 53: 41$ & 1.4398 \\
\cline { 2 - 4 } & $2014 / 04 / 12$ & $01: 59: 14 \sim 02: 51: 28$ & 4.2437 \\
\hline \multirow{2}{*}{$\begin{array}{l}\text { DATA3: } \\
\text { Different month } \\
\text { or different time }\end{array}$} & $2015 / 03 / 14$ & $13: 20: 25 \sim 14: 12: 28$ & 2.4321 \\
\cline { 2 - 4 } & $2015 / 09 / 26$ & $02: 02: 06 \sim 02: 54: 18$ & 2.8373 \\
\hline
\end{tabular}

From Table 3.2, the mean RMSE is $5.7236 \mathrm{~cm} / \mathrm{s}$. It is obvious that the retrieval accuracy by using two input parameters $\left(\sigma_{K u}^{0}, s w h_{k u}\right)$ is much better than only one input parameter $\left(\sigma_{K u}^{0}\right)$. A conclusion can be obtained that introducing $s w h_{k u}$ is usefull to improve the wind speed retrieval accuracy.

\subsection{The Multi-Parameter Wind Speed Retrieval}

In this section we use four parameters $\left(\sigma_{K u}^{0}, \sigma_{C}^{0}, s w h_{k u}\right.$ and $\left.s w h_{C}\right)$ as input parameters and wind speed as output parameter to establish network model. We choose the data in period (00:39:07 01:31:12) of 2015/03/01 and use the first 1000 sets of data as training samples and the rest as testing samples.

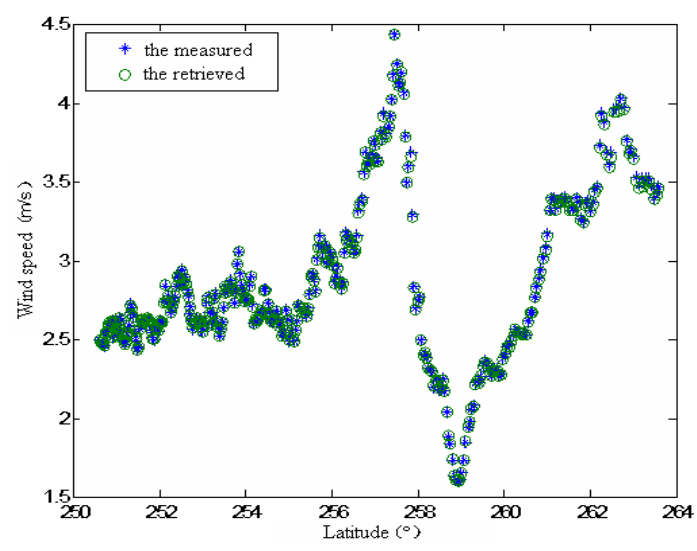

Figure3.4 Measurements and retrieval values

Similarly, we have also used some data from other time periods to do the retrieval. The results are shown in table 3.3. We compare the tested and measured values by calculating RMSE. We can obtain the mean $\mathrm{RMSE}=4.9723 \mathrm{~cm} / \mathrm{s}$ as shown in figure 3.4 .

We can draw a conclusion from Table 3.3 and figure 3.2-3.4. A four-parameter wind speed retrieval method has a better accuracy than a one or two parameter retrieval method does, and the sea surface wind speed is relative to many factors such as $\sigma_{r}^{0}, s w h_{k n}$ and $s w h_{r}$, besides $\sigma_{K u}^{0}$. The further efforts are needed to prove the conclusion by the relative buoy data in the future.

Table 3.3 The root mean square error value for other test data 


\begin{tabular}{|c|c|c|c|c|}
\hline training sample & \multicolumn{4}{|c|}{ 2015/03/01 00:39:07 01:31:12 } \\
\hline & \multicolumn{2}{|c|}{ test data } & \multicolumn{2}{|c|}{ rmse $(\mathrm{cm} / \mathrm{s})$} \\
\hline & & & Multi-parameters & Dual-parameters \\
\hline $\begin{array}{l}\text { DATA1: } \\
\text { The same time }\end{array}$ & $2015 / 03 / 01$ & 00:39:07 01:31:12 & 0.4767 & 0.4778 \\
\hline \multirow{3}{*}{$\begin{array}{l}\text { DATA2: } \\
\text { Different time }\end{array}$} & $2015 / 03 / 14$ & 02:01:17 02:53:12 & 1.2469 & 1.3525 \\
\hline & $2015 / 04 / 25$ & 02:01:29 02:53:41 & 1.4191 & 1.4398 \\
\hline & $2014 / 04 / 12$ & 01:59:14 02:51:28 & 3.7212 & 4.2437 \\
\hline \multirow{3}{*}{$\begin{array}{l}\text { DATA3: } \\
\text { Different } \\
\text { month or } \\
\text { different time }\end{array}$} & $2015 / 03 / 14$ & $13: 20: 25 \sim 14: 12: 28$ & 1.0227 & 2.4321 \\
\hline & $2015 / 09 / 26$ & 02:02:06 02:54:18 & 2.8826 & 2.8373 \\
\hline & $2015 / 12 / 25$ & 02:02:18 02:54:11 & 19.5414 & 22.0362 \\
\hline
\end{tabular}

\section{Conclusion}

For HY2 altimeter, the measured wind speed is not only relative to $\sigma_{K u}^{0}$, but also to $\sigma_{C}^{0}, s w h_{k u}$ and $s w h_{C}$. Introducing the other three parameters $\left(\sigma_{C}, s w h_{k u}\right.$ and $\left.s w h_{C}\right)$, especially $s w h_{k u}$, can greatly improve the wind speed retrieval accuracy. Neural network algorithm is an alternative method of retrieving wind speed, especially the sea surface wind speed inversion accuracy has a relatively large improvement by introducing $\sigma_{C}^{0}, s w h_{k u}$ and $s w h_{C}$. The neural network is suitable for HY2 altimeter wind speed retrieval because it has a strong learning ability and generalization ability.

Acknowledgment: The project is supported by National Natural Science Foundation of China (No. 41376181, No.41676090)

\section{References}

1. Yijun $\mathrm{He}, \mathrm{Ge}$ Chen at al, "The research and application of altimeter ocean remote sensing" $[\mathrm{M}]$, Beijing:Science Press, 2002.

2. Xingwei Jiang, Mingsen Lin, Jianqiang Liu, The HY-2 satellite and its preliminary assessment[J],International Journal of Digital Earth Vol.5,Iss.3,2012.

3. Li Shuiqing, Zhou Liangming, Li Zhangbin et al, "A Physics-Based Dual-Frequency Approach for Altimeter Wind Speed Retrieval", J. Ocean Univ. China(Oceanic and Coastal Sea Research), Doi 10.1007/s11802-014-2335-9,2014, Vol.13,No.4, pp.579-583.

4. Ren Lin,Yang Jingsong,Zheng Gang,Wang Juan, Chen Peng, "Wave effects on the retrieved wind field from the advanced scatterometer(ASCAT)", Acta Oceanol.sin., 2015,Vol.34,No.1, pp.79-84.

5. Jiasheng Tian; Qiaoyun Liu; Wan Pan; Jian Shi; "Improved algorithm of retrieving oceanic surface wind speed based on a stratified media model at high sea states", IET Microwaves, Antennas \& Propagation, Volume 8, Issue 15, 09 December 2014. pp.1333-1339.

6. Wang Jin, Zhang Jie, Fan Chenqing andWang Jing, "A new algorithm for sea-surface wind-speed retrieval based on the L-band radiometer onboard Aquarius", Chinese Journal of Oceanology and Limnology, 2015, Vol.33, No.5, pp.1115-1123.

7. William, J, Blackwell, Frederick, W, Chen, Neural Networks in Atmospheric Remote Sensing[M], Artech House; 1 edition (April 30, 2009),

8. Haynes G S. Radar altimeter mean return waveforms from near-normal-incidence ocean surf ace scattering [J]. IEEE Trans. Antennas Propagation, 1980, 28:687-692.

9. Mohamed, A, Mohandes, Shafiqur, A Neural Networks Approach for Wind Speed Prediction [J]. Renewable Energy, 1998, 13(3): 345-354. 